\title{
Open Science, but Correctly! \\ Lessons from the Heinsberg Study
}

\section{A Comment}

Authors (alphabetically, by contribution)*

Concept and initiation:

Caroline Fischer, ORCID iD: 0000-0001-5870-4498, University of Potsdam

Jo Havemann, 0000-0002- 6157-1494, Access 2 Perspectives

Tamara Heck, 0000-0001-5519-6395, Leibniz Institute for Educational Research and Educational Information

Hans Henning Stutz, 0000-0003-3360-1243, Aarhus University, Department of Engineering First draft:

Nate Breznau, 0000-0003-4983-3137, University of Bremen

Tamara Heck, 0000-0001-5519-6395, Leibniz Institute for Educational Research and Information

Katja Mayer, 8000003-1184-595X, University Vienna

Isabella Peters, 0000-0001-5840-0806, Leibniz Information Center for Economics \& Christian-

Albrechts-University in Kiel

Philipp Schrögel, 00000003-0892-8703, Karlsruhe Institute of Technology

Revisions:

Nate Breznau, 0000-0003-4983-3137, University of Bremen

Caroline Fischer, 00000001-5870-4498, University of Potsdam

Jo Havemann, 00000000-6157-1494, Access 2 Perspectives

Tamara Heck, 8000001-5519-6395, Leibniz Institute for educational research and educational information

Katja Mayer, 00000003-1184-595X, University of Vienna

Isabella Peters, 00000001-5840-0806, Leibniz Information Center for Economics \& ChristianAlbrechts-University in Kiel

Philipp Schrögel, 00000003-0892-8703, Karlsruhe Institute of Technology

Hans Henning Stutz, 8000003-3360-1243, Aarhus University, Department of Engineering

* This is an English translation of the German original, "Open Science, aber richtig! Was wir aus der Heinsberg-Studie lernen können" posted on MetaArXiv. The authors are open science fellows from the Wikimedia Freies Wissen ('Free Knowledge') Fellow Program (cohort 2019/2020). The idea for this paper sprang from this research community. The authors would like to thank the other Free Knowledge Fellows for numerous comments and feedback on the contribution. 


\section{Introduction}

During the Novel Coronavirus Pandemic, the call for access to academic knowledge, publicly available data and studies, and transparent communication is growing louder. In other words, 'open science' is ringing in the ears of the public debates surrounding the pandemic. However, there is a tension between opening science and following best practices in crisis management. Herein we investigate this tension and concomitant challenges to good open science practice, reviewing both criticisms and opportunities for a new understanding of science.

The pandemic leads to more public attention, if not demand, for timely communication of relevant research results. People look for knowledge to reduce the uncertainty in their behavioral decisions. In Germany, scientific processes and results have been discussed in the media like never before. Two studies in particular are exemplary: The so-called "Heinsberg Study" led by Hendrik Streeck and the "Charité Viral Load in Children Study" by the virologist Christian Drosten. The findings of these studies were both welcomed and criticized, many different sides questioned their scientific quality and informative value. However, they also sparked negative reactions because of the manner in which their preliminary results were presented and communicated (see report from $\underline{B R}$ and an interview with Drosten) ${ }^{1}$.

In crisis situations, the general tension between the speed of dissemination and the quality of scientific knowledge becomes particularly apparent. Central principles of open science are the greatest possible transparency and opening up knowledge to a broad discourse.

So what can we learn from the debates about scientific integrity in times of crisis for good open science practice? How can we act as openly as possible, but be careful with public expectations?

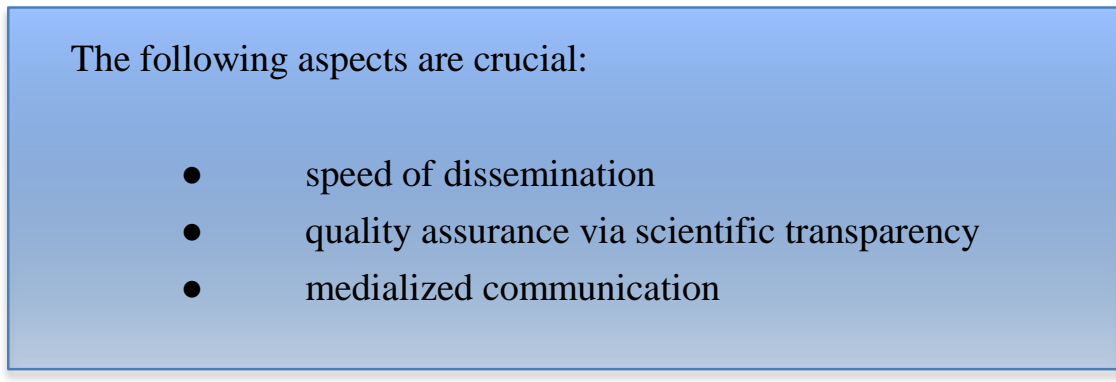

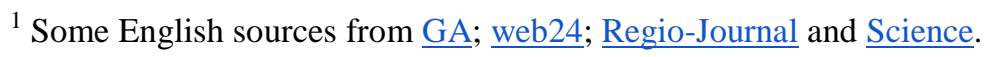




\section{The Heinsberg Study in the context of open science}

On April 9th, 2020, the virologist Hendrik Streeck and colleagues presented interim results of the Heinsberg Study at a press conference. This presentation took place before a manuscript or the research data were published. It was only in early May that a first preprint article was uploaded to medRxiv, later updated in early June. The preprint states that the research data can be obtained on request. This is common practice in health research because sensitive personal data could cause harm to the participants if broadcast in public. There is currently (as of 02.07.2020) no peer review of the study available. In the public sphere, other scientists criticized that the researchers "only" had interim results and these were presented for the first time at a national press conference.

Open science requires the consistent implementation of good scientific practice. It also has the goal of doing science even more than before as a community. Therefore, the guiding principle of the Open Science Movement is that the scientific processes - from ideas, methods, analyses and data, to results should be published as early as possible. The principle is deliberately not 'publishing as fast as possible', but 'sharing knowledge as early as possible'.

Earliest possible publication of scientific processes generates an informed public. This promotes discussion, which in turn leads to an improvement in research. It should be borne in mind that scientific knowledge is always associated with a certain degree of uncertainty and provisionality, meaning that specialist commentary is especially important to guide deliberations and public understanding of 'facts'. When knowledge is discussed and critically reviewed during the developmental process, it maximizes scientific utility. In times of crisis it is a waste of time (and potentially human lives) to endure the severalyear waiting time to publish in an academic journal as a form of quality control. Thus, COVID-19 researchers follow open science principles and publish their data and results as quickly as possible. Research data and scientific manuscripts are shared and collected on collaborative online platforms for SARS-CoV-2 and COVID-19 data.

The researchers in the Heinsberg Study also wanted to share their findings quickly (interim results) and broadly (large-scale press conference). Nevertheless, there was criticism of this approach, much of which we believe to be justified.

\section{Speed of dissemination (vs. quality)}

According to one estimate, the literature published on the pandemic since January 2020 comprises more than 25,000 manuscripts, with the amount doubling every 20 days. The public is witnessing one of the greatest explosions of scientific literature in recent times.

So-called preprint servers or repositories such as preprints.org, OSF preprints, bioRxiv or medRxiv - enable publication at a speed that is not possible in print-driven publishing practices. Many research 
funders are also currently supporting accelerated publication processes. The rapid dissemination of results or intermediate results is desirable from an open science perspective, if they meet transparency requirements to enable peer review by peers. This means that knowledge that has been validated without a detailed assessment process can serve as an information basis for other researchers, stimulating further research, avoiding redundancies and providing policymakers with evidence on which to make sound policies.

However, too much uncertainty and scientific mistakes in interim results, can also cause great damage, especially if politically relevant decisions are based on them. Some platforms such as bioRxiv and medRxiv adapted to this by introducing some new and more intensive checking of uploads, especially when it comes to medical research that could have an impact on health treatments or that involve complicated statistical analyses.

In traditional peer review procedures, experts review the research methods, data and results of their colleagues before the final results are published. Interim results are also normally discussed at scientific conferences where they go through an early form of peer review. After a structured peer review in the publication process, the results are published in a scientific journal or book for example. With peer review, science has established an important instrument for testing research methods, analyses and results - even if the traditional peer review process has its weaknesses (see: Rödel, 2020; interview with Ross-Hellauer).

Most publications have not previously been peer reviewed on preprint servers. As the review process can take so long, this makes results available to other researchers faster and knowledge dissemination proceeds immediately. This means the only 'peer review' comes post-preprint in the later publication process. If one considers that (a) preprints are a tool that ensures compliance with the principle of the earliest possible publication and (b) classic pre-publication peer review is a tool to ensure the principle of quality assurance, it becomes clear that both principles cannot comfortably co-exist.

Nevertheless, it should be emphasized that preprint manuscripts can be read and understood directly by scientists and can be checked so long as research data are made transparent. This did not happen in the Heinsberg Study. However, Hendrik Streeck's defending statement that a preprint publication would have taken 2 to 3 weeks cannot be dismissed entirely, since preprint servers are sometimes overloaded with inquiries in the current situation. Moreover, research data should also be processed accordingly for other researchers, for example removing personal identifiers, which in turn takes time. In the sense of open science, however, both aspects are important to ensure transparency, traceability and verifiability of research. These are ethical points that should not be neglected in the principle of the earliest possible publication.

Now we see in a pandemic that conferences do not take place or no longer take place to a sufficient extent; meaning this form of initial peer review is not available. Moreover, waiting for a conference presentation also does not work when quick, if not immediate, feedback from colleagues is required before 
releasing results of a study. So what can scientists do in the future to quickly publish research results and still check their quality?

An additional quality assurance mechanism would be conceivable, such as an expert advisory board that forms and is available to give feedback - organized to the point that it can easily be called upon and channel calls to the experts. For this purpose, specialist groups or overarching organizations, such as the German Science Foundation, could form a pool of scientists who, similar to the formal peer review, can be requested for short-term assessments. Anyone who applies for and implements third-party funded projects knows that such a body is often convened. Such solutions should also be used for contract research if it is as important as the Heinsberg Study was; i.e., a study that could determine policies taken to reduce (or inadvertently increase) the likelihood of infection and thus death for an entire society.

In the sense of an open peer review, assessments by such an organized specialist group should also be published in order to make quality assurance measures transparent.

\section{Quality assurance: Open data as a "must"}

Whatever it takes, data must be shared. Without data, the study could be entirely fabricated. According to the principle of FAIR - findable, accessible, interoperable, reusable - open science must always strive for the greatest possible transparency and verifiability of the research process. It is only when preliminary and final research data, methods, protocols and standards are shared that the results can be checked at all. This is particularly important if the results have not yet been validated through a peer review process, but is nonetheless always desirable.

In the case of the Heinsberg Study, the data was not available at the time of the press conference, so a quick assessment of its quality was not possible. This is not in line with the idea of open science and should be criticized. Complete transparency requires more time from researchers thus better technical solutions need to be implemented, such as preparing a template that anonymizes data during the collection process. This is a challenge that must be addressed in the future.

The criticism of the lack of open or partially open data is currently not only directed at science. The public sector is also required to collect and make available relevant information as quickly as possible while maintaining data protection. The crisis shows how little the necessary protocols and infrastructures are still developed and that there is a great need for action. Science rightly criticizes the poor quality and lack of traceability of accessible health data and their contextual information - this data is often not FAIR. The connectivity and interoperability that are so important for public health issues cannot be guaranteed in this way. 


\section{Medialized communication}

Open science also affects the way in which scientific knowledge is communicated to and among diverse publics. In addition to traditional scientific publication formats such as science magazines, this also applies to mass media and a digitalized public sphere.

Open communication and simultaneous quality checks work reasonably well within science networks. A good example is the aforementioned Charité Viral Load in Children Study by Drosten. It was published as a preprint version, and because of this transparency other researchers drew attention to errors it likely contained. Both sides exchanged views and the results of the study were revised. This process is common in science and is part of the peer review process, or better put: it defines the scientific process itself. What is new, however, is that open science is expanding the circle of actors, because scientific discussions are now being held in open formats that are accessible to everyone (e.g. via tweets on Twitter).

But not only the new communication formats are a challenge. Favored by the already existing social and political debates, the scientific work on the Novel Coronavirus in Germany has been touted in some media as an interpersonal conflict between two competing heroes - Prof. Drosten and Prof. Streeck

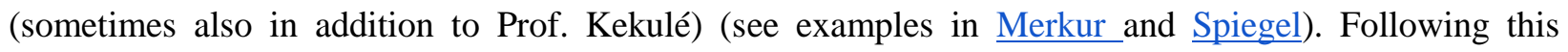
narrative, the mass media reporting on the Heinsberg Study also focused on the conflict (see also the opinion of Dieter Lenzen, President of the University of Hamburg).

Other factors with a view to the actors make the press release of the Heinsberg Study a special event in many ways. The situation was exacerbated by the fact that NRW Governor Laschet, who commissioned the study, had previously taken a pointed political position and was probably waiting for, or at least hoped for, certain scientific results in favor of reopening quickly. Interestingly, Hendrik Streeck had also been critical of the Novel Coronavirus as not as deadly or infectious as many were saying. In addition, these events were remarkable in comparison to typical scientific undertakings because the results were 'published' in a press conference with the Prime Minister, accompanied by a PR campaign by the professional agency "Storymachine" with an explicit position in favor of easing lockdown measures.

The accusation of "politically motivated contract research" is evident from these events, but the evidence is anecdotal for now. It should be noted that research is never devoid of motives, even supposedly independent and objective research. Contract research is perhaps more dangerous, or susceptible to unobjective manipulation, but in and of itself is not a problem if open science is always followed. Of course, it must be a research contract and not, a results contract as is the ostensible story that unfolded here. Our conclusion, regardless of the motives, is the same: the scientific processes, methods and motivations must be transparently verifiable. If these actions were taken, the Heinsberg Study would have been insulated from the problems it and its unusual PR campaign created. 
The journalistic and public response here makes clear the need for more sensitivity to media staging. Otherwise, the problem of "science by press conference" emerges, a problem behind widespread acceptance of pseudo-scientific claims such as Andrew Wakefield's false 1998 claim that vaccination triggers autism or Gilles-Éric Séralini's 2012 study claiming genetically modified plants cause cancer. The pandemic situation certainly created a political urgency, but a careful communication style must be chosen, especially when it comes to public health issues

Thanks to digital technologies, today's science has more opportunities to communicate research processes and results and to let those interested directly participate in these processes. Researchers publish open access versions of their work and more often write blog posts that anyone can read; in addition they, or those speaking on their behalf, are active on social media (such as the Twitter account specially created for the Heinsberg Study). These new channels and formats not only have the potential to communicate processes faster and more broadly, they also facilitate access to science. This breaks down the previously delimited, silo-like, internal scientific discourse publicly accessible on the one hand, but it becomes vulnerable to various non-scientific attacks and misuses on the other. New challenges are the incorrect or deliberately misleading, politically motivated media presentations of scientific knowledge. Research, politics and the media must embrace the new digital world, but find ways that it does not cause chaos or unnecessary human death or harm. Reliance on for-profit PR agencies or political decision-makers who want to act based on evidence but also on their own-political-career interests is dangerous. Instead, the competencies of all involved actors need to be expanded, how to communicate uncertainties, how to provide the knowledge relevant for the results so that it remains verifiable, and how to maintain a scientific debate culture, especially in times of crisis, without being instrumentalized or weaponized for nefarious interests. 


\section{Corrections to the Handling of the Heinsberg Study}

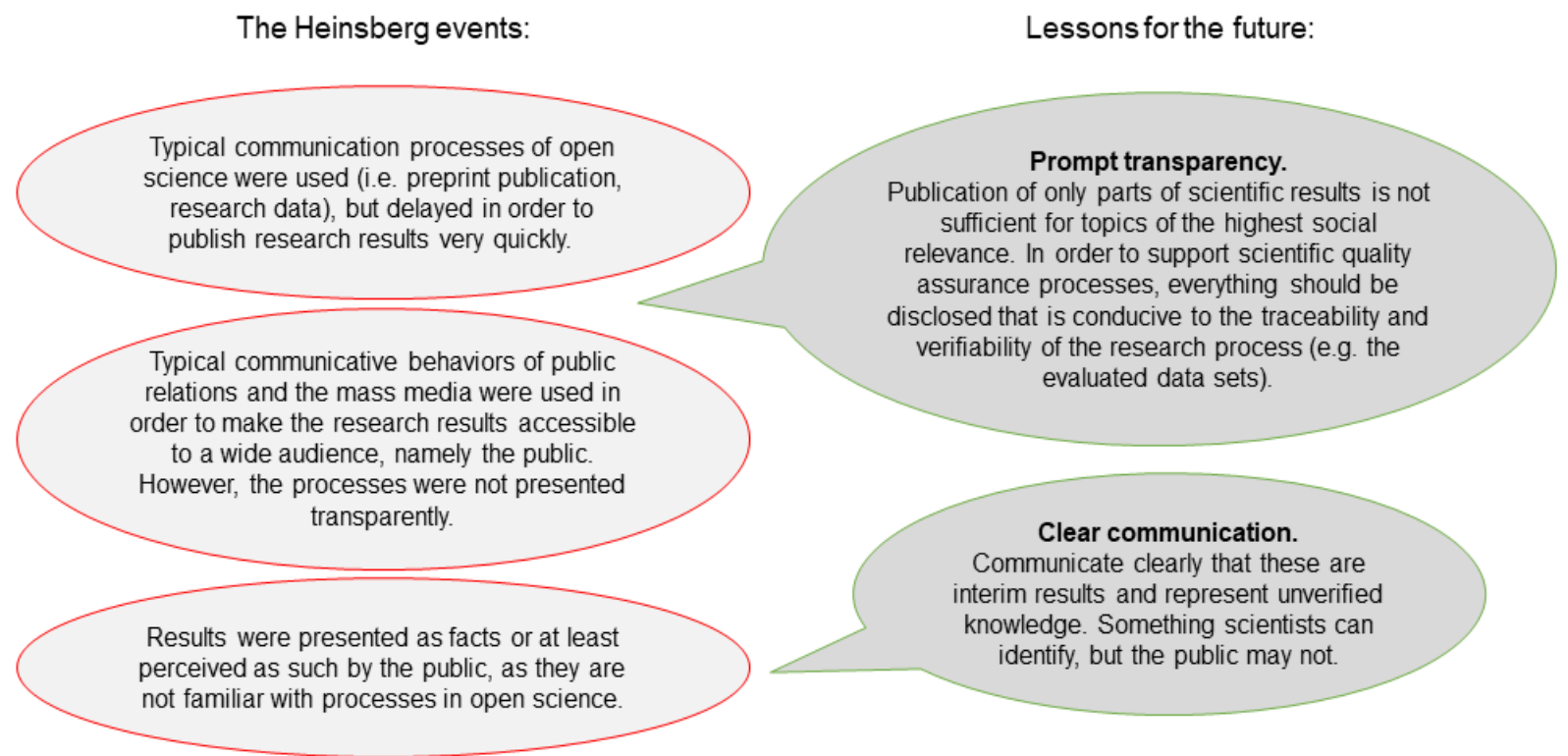

Fig 1. Events and lessons from the Heinsberg Study

\section{Lessons Learned}

We derive five lessons from the events of the Heinsberg Study that underline the importance of open science and suitable forms of communication in societal-crisis situations. They are an appeal to science and the public to cooperate better in the future in the field of communication. The goal should be to increase the intersection of knowledge, expectations and needs between science and the public. We see open science not only as a helpful, but also an indispensable tool for realizing this outcome.

\section{(1) No shortcuts: Scientific communication takes care and time}

Especially in crisis situations and on issues that affect health and other aspects of immediate social relevance, special care must be taken in the communication of research. Scientific knowledge and communication processes cannot be accelerated infinitely, they always take a certain amount of time, e.g. in scientific peer review, in the detailed documentation of the procedure, in data cleansing or in communicating results.

The classification and presentation must also be carefully chosen. In medical journalism there are certain established criteria from which to draw. Sufficient time must be allowed for this. Time that is often not allowed in the communication process because the need for answers is putting too much pressure on 
politicians and the public. It is only through sufficient time and special care that knowledge can be meaningfully developed and applied to decision making.

\section{(2) Results vs. Decisions: Clear communication of expectations needed}

Researchers are often not used to communicating directly with the public. Communication within disciplines follows scientific-cultural patterns that researchers acquire during their studies and professional life. Within the disciplines, the expectations of the researchers and the communication processes are clear. However, this changes when communication is interdisciplinary, i.e. if at least two disciplines are involved. Such research projects spend a lot of time negotiating and making clear the expectations of each other and the respective disciplines.

The communication gap between disciplines is a canyon between scientists and the public. Only in exceptional cases is there negotiation and communication processes between science and the public. Open science has to deal with, and learn from, inquiries and criticism from the public. This requires both communication partners to get involved and work together on how such scientific communication should look. This also includes that science explains how research results are to be classified, what it can and cannot do, and what can be done with remaining uncertainties. To do this, however, science should not come under fire from the public or even be politically exploited; instead, space and time should be given for the knowledge and quality assurance processes.

\section{(3) Missing formats: further development and use of suitable forms of communication}

The previous formats of scientific communication (e.g. journalistic preparation of the research results), internal science communication (e.g. journal articles) and public relations (e.g. press conferences) do not realize the requirements for careful and publicly-awaited communications. In the sense of open science, it is particularly advisable to (further) develop digital formats, e.g. platforms such as https://outbreaksci.prereview.org/ or https://prereview.org/, which have so far been more for internal science exchange. These can and should be used to open the quality assurance process. However, such platforms do not yet meet all the expectations of the public as their consumption of science is radically different from scientists.

The problem associated with the interpretation of the research results (among the public and policymakers) in the face of uncertain knowledge and complex relationships can also be addressed by means of suitable communication formats. Here, it seems, our society lacks awareness that scientific information does not always have "final" results, but the publication of which are part of an important process of transparency, further review and the construction of knowledge. The interim results are not the real problem, but their handling and communication. Here, the open science community should do more to 
bring society closer to the awareness of science as a dynamic process (see for example the piece by Mafalda Sandrini and Kata Katz).

As previously suggested, new forms of institutionalized open science should be adopted to improve reliability of results prior to their communication. For example, a network of rapid-response scientists to be dispatched in emergencies to review studies of immediate health and policy impact.

(4) Non-transparency creates doubts: Rapid opening of critical components of the scientific knowledge process

It cannot be mentioned often enough that the more quickly it can be used and subjected to a critical examination, the more quickly it can be used to make provisional scientific knowledge. For this purpose, all critical components of the scientific knowledge process, i.e. everything that is useful for the assessment of a research result, must be made available transparently. This is the core goal of open science and also fundamental to the fight against the Novel Coronavirus Pandemic. For example, the open sharing of gene sequences of the virus enabled rapid development of antibody tests.

\section{(5) Robust knowledge emerges together}

A transparent 'traceability' of the parties involved in the knowledge and quality assurance process also makes it clear that science is never a result of individual activities, but is always a collective and, at best, collaborative enterprise. If 'groundbreaking' scientific results are only presented by a research team or detached from the scientific discussion, then one should be careful in their interpretation. Scientific results are in a complex connection with previous and future knowledge production. What is sometimes declared in the media as a "dispute" among researchers is a necessary process of securing knowledge and is based on the principle of peer review. Scientific results can only be interpreted within this context and can become secure knowledge only through collaboration.

Science and scientific progress are fundamentally dependent on the sharing of knowledge, i.e. open science. In crisis situations, traditional formats such as articles in specialist magazines are too slow, so other formats must be fostered (see Lesson 3). Especially in times of crisis, shared knowledge should be treated as a common good. It is clear to us that Lesson 1 and Lesson 4 are in a certain tension, but we are certain that a consistent implementation of well-thought-out open science practices will make these tensions productive. 